\title{
Timing Phase Offset Recovery Based on Dispersion Minimization
}

\author{
Wonzoo Chung, William A. Sethares, and C. Richard Johnson, Jr., Fellow, IEEE
}

\begin{abstract}
This paper presents a blind timing phase offset recovery scheme that attempts to optimize the baud spaced equalizer output mean square error (MSE) for a realistic equalizer length that is usually shorter than the ideal length. Among the existing blind timing recovery schemes, few are designed for equalizer output MSE optimization, and none are designed for the realistic case when the equalizer is short. The proposed algorithm (that is based on a cost function that minimizes the dispersion of the received signal) attempts to minimize the MSE of a one-tap equalizer output. It also exhibits good performance for relatively short equalizers. Conditions for the unimodality of the dispersion minimization cost are investigated, and a geometric relationship to the minimum MSE (MMSE) timing offset is shown qualitatively. The detailed MSE performance of the algorithm is investigated for the representing classes of channels by comparing existing blind timing offset estimation schemes.
\end{abstract}

Index Terms-Adaptive blind synchronization, MMSE timing offset, timing offset recovery.

\section{INTRODUCTION}

$\mathbf{I}$ $\mathrm{N}$ most digital communication systems, digital sources are conveyed via excess bandwidth analog pulses that are designed in satisfaction of the Nyquist Criterion [1] to prevent aliasing for a proper sampling offset in the absence of a multipath channel. Due to aliasing, the performance of the receiver with a symbol-spaced equalizer is sensitive to the sampling offset, especially in the presence of multipath interference, where there is (in general) no sampling offset that can perfectly eradicate aliasing. Furthermore, for the scenario where multisensor outputs are combined in order to increase SNR, a proper sampling offset for each sensor can contribute to significant performance improvement [2]. In both cases, estimation of an optimal timing offset is an important task, but the criterion for "optimality" is not obvious when the timing offsets must be determined at the synchronization stage. A natural approach is to minimize the overall MSE performance at the input to the detection device, i.e., to minimize the MSE

Manuscript received November 14, 2003; revised February 12, 2004. W. Chung and C. R. Johnson, Jr. were supported in part by the National Science Foundation under Grant ECS-9811297 and NxtWave Communications (now ATI). The associate editor coordinating the review of this manuscript and approving it for publication was Prof. Gregori Vazquez.

W. Chung is with Dotcast Inc., Kent, WA 98032 (e-mail: wchung@dotcast.com)

W. A. Sethares is with the Department of Electrical and Computer Engineering, University of Wisconsin, Madison, WI 53706 USA (e-mail: sethares@ece.wisc.edu).

C. R. Johnson, Jr. is with the School of Electrical and Computer Engineering, Cornell University, Ithaca, NY 14853 USA (e-mail: johnson@ece.cornell.edu). Digital Object Identifier 10.1109/TSP.2004.842211 at the output of the equalizer [3]. Unfortunately, direct estimation of the timing offset that minimizes the overall MSE requires knowledge of the analog channel, and the procedure is computationally complex. Adaptive approaches based on training sequences or decisions from detection devices can be used in place of direct computation, but directly coupling the updates of the timing offset and the equalizer parameters is considered undesirable in actual implementation for a variety of reasons [4]. Since reliable reference or decision data is often unavailable during the acquisition phase, the estimation of the synchronization parameters benefits from the use of blind (nondata aided) methods. In order to avoid the sensitivity of sampling offset in symbol spaced processing, sampling above the Nyquist rate of the analog pulses (so-called fractionally sampling) may also be used [5]. However, fractionally spaced sampling and equalization is not always feasible, especially when the additional hardware requirements cannot be met. For example, in digital audio/video broadcasting, the signals are often transmitted over a channel with delay spread that is too long to be equalized with a fractionally spaced equalizer using a restricted number of taps [6].

Therefore, a desirable timing offset estimation scheme for a symbol spaced receiver should i) be blind, ii) not rely on the equalizer output, and, at the same time, iii) optimize the MSE of the equalizer output.

Among the existing schemes for timing phase offset recovery, the output energy maximization (OEM) method comes closest to satisfying these three conditions. The algorithm finds a timing offset that maximizes the sampler output energy and consequently maximizes SNR. In [4], it was shown that maximizing SNR is approximately equivalent to minimizing the overall MSE of the receiver when equipped with an infinite length equalizer. In practice, however, the equalizer length is always constrained. When the equalizer is short, the timing offset at that the SNR is maximized may be significantly different from the timing offset at that the MSE is minimized. Thus, OEM may not near optimal for a short equalizer.

This paper examines an alternative blind approach to timing offset estimation (see also [7]) that attempts to minimize MSE for a short equalizer, as OEM attempts to optimize MSE for a sufficiently long equalizer. The alternative timing offset estimation is designed to choose a timing offset minimizing the dispersion of the sampler output. Simulation results in [2] and [7] suggest that the MSE performance of this dispersion minimization timing offset estimation method is improved over the OEM method for the practical case when the equalizer length is short. This paper presents an analysis of the dispersion minimization 


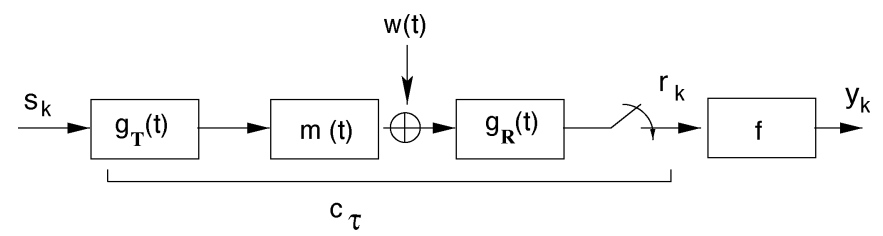

Fig. 1. Digital communication systems model.

timing offset as an approximation of the minimum mean square error (MMSE) timing offset for a one-tap equalizer.

Section II defines the optimal timing offset as a timing offset minimizing MSE of the equalizer output. An existing blind timing algorithm (OEM-timing) that is optimized for infinite length equalizers is compared with a training-based timing algorithm optimized for a short length (one-tap) equalizer. The importance of a timing offset optimized for the one-tap equalizer is emphasized.

Section III proposes a blind adaptive approach for the timing offset that attempts to minimize the MSE of the one-tap equalizer output (called DM-timing) and analyzes its cost function. Conditions under which the timing estimation is unimodal are derived, and a geometrical interpretation of the relation between DM-timing and OEM-timing is presented.

Section IV investigates how well this DM-timing offset approximates the MMSE timing offset for a one-tap equalizer. Due to the complexity of the closed-form analysis for DM-timing cost function of a general channel, quantitative analysis is performed for the representative classes of channels, pure delays channels, clustered channels, and sparse channels. The investigation and simulation results show that DM-timing offset quite well approximates MMSE timing offset optimized for the MMSE one-tap equalizer for mild multipath channel and always performs better than OEM timing offset for the one-tap MMSE equalizer. The final section concludes the paper.

\section{LINEAR EQUALIZER OUTPUT MSE AND TIMING}

This section reviews the problem of timing offset in digital communication systems and defines the optimal timing offset with respect to the equalizer output MSE. Consider a digital baseband communication system in Fig. 1, where the digital sources $\left\{s_{i}\right\}$ drawn from a finite alphabet set are converted to an analog signal $s(t)$ by convolving with the pulse shape function $g_{T}(t)$

$$
s(t)=\sum_{i=-\infty}^{\infty} s_{i} g_{T}(t-i T)
$$

where $T$ is referred to as the symbol period. The transmitted signal is subject to distortion due to multipath and noise. The multipath is characterized by a finite impulse response $m(t)$

$$
m(t)=\sum_{i=0}^{N_{c}-1} \rho_{i} \delta\left(t-\tau_{i}\right)
$$

where $\tau_{i}$ and $\rho_{i}$ denote the delay and the complex path gain of the $i$ th path, respectively. The noise is modeled as a wide sense stationary (w.s.s.) zero mean additive white Gaussian process $w(t)$ with variance $\sigma_{w}^{2}$ that is uncorrelated with the signal $s(t)$. At the receiver, the received signal is processed by a matched filter $g_{R}(t)$ with output $r(t)$ that can be written

$$
r(t)=\sum_{i=-\infty}^{\infty} s_{i} c(t-i T)+w(t) \star g_{R}(t)
$$

where the analog channel $c(t)$ is

$$
c(t)=g_{T}(t) \star m(t) \star g_{R}(t)
$$

and where $\star$ denotes the convolution operator. The matched filter output is sampled at the symbol rate $1 / T$ with a timing offset $\tau(0 \leq \tau<T)$.

$r(k T+\tau)=\sum_{i=-\infty}^{\infty} s_{i} c((k-i) T+\tau)+\left.w(t) \star g_{R}(t)\right|_{t=k T+\tau}$

For practical reasons, the pulse $g_{T}$ has excess bandwidth (i.e., the maximum frequency in $g_{T}$ is larger than $1 / T$ ). However, $g_{T} \star g_{R}$ is designed under the Nyquist criterion [1] so that the sampled signal does not have aliasing for the perfect timing offset in the absence of a multipath channel.

To examine the effect of the timing offset on the sampled signal in the presence of a multipath channel, assume that the received filter is matched to the pulse shape $g_{T}(t)=g_{R}(-t)$ and that the pulse shape is symmetric $\left(g_{R}(t)=g_{R}(-t)\right)$ (for example, a square root raised cosine filter [1]). Then, the sampled noise component $\left\{\left.w(t) \star g_{R}(t)\right|_{t=k T+\tau}\right\}$, which is denoted by $\{w[k]\}$, becomes a white zero mean Gaussian process with variance $\sigma_{w}^{2}$ independent of $\tau$ from the property of the symmetric Nyquist filter $g_{R}(t)$ and the whiteness of $\{w(t)\}$. On the other hand, the signal component is dependent on the timing offset. By defining the sampled channel with respect to the sampling offset $\tau$ as a vector $\mathrm{c}_{\tau}$, i.e.,

$$
\mathrm{c}_{\tau}[k]:=c(k T+\tau)
$$

the sampled output can be written in the discrete time domain as

$$
\mathrm{r}=\mathrm{c}_{\tau} \star \mathrm{s}+\mathrm{w} .
$$

In the absence of multipath, $\mathrm{c}_{\tau}[k]$ is given by

$$
\mathrm{c}_{\tau}[k]=g_{T} \star g_{R}(k T+\tau)=\delta\left(k+\frac{\tau}{T}\right)
$$

and thus, the correct timing offset $\tau=0$ perfectly restores the digital data $\left\{s_{k}\right\}$. In the presence of multipath, $\mathrm{c}_{\tau}$ cannot be reduced to a single nonzero tap in general. Furthermore, the aliasing due to excess bandwidth of $g_{T} \star g_{R}$ can deepen the spectral null of the sampled channel $\mathrm{c}_{\tau}$, depending on the timing offset $\tau$, and can effect the MSE performance of the equalizer [8]. Thus, in the presence of multipath, it is desirable to determine jointly the timing offset and the equalizer.

Consider an MMSE equalizer $f_{\tau}$ for a given channel $\mathrm{c}_{\tau}$ with respect to a given timing $\tau$. The optimal timing offset $\tau_{\text {opt }}$ is 
to minimize the MSE of the equalizer $f_{\tau}$ output $y(\tau)$ in the presence of a multipath channel, i.e.,

$$
\tau_{\text {opt }}=\arg \min _{\tau} E\left\{\left(y_{k}(\tau)-s_{k+d}\right)^{2}\right\}
$$

where the delay $d$ applied to the source (or training) sequence is called the system delay. This definition of timing offset optimality can be extended to the problem of combined multiantenna inputs. In this form, determining the optimal timing offset can be viewed as a nonlinear quadratic optimization problem over the channel vectors under a constraint imposed by the parameter $\tau$. This problem is difficult because a direct update for the timing offset based on feedback from the equalizer output is undesirable not only from an implementation point of view [4] but also due to the latency caused by the convergence of the equalizer. Furthermore, a closed-form estimation of the optimal timing offset is infeasible due to the extremely complicated nonlinear relation between the parameter $\tau$ and the resulting sampled channel $\mathrm{c}_{\tau}$.

However, relatively simple solutions exist for the extreme cases of equalizer length; for a one-tap equalizer, $\left(N_{f}=1\right)$, and for an infinite length equalizer, $\left(N_{f}=\infty\right)$. For the MMSE equalizer of length $N_{f}=1, \infty$, the MMSE with respect to the system delay $d$ and timing $\tau$ is given by

$$
\begin{aligned}
\operatorname{MMSE}\left(d, \tau, N_{f}=1\right) & =1-\frac{\left|\mathrm{c}_{\tau}[d]\right|^{2}}{\left\|\mathrm{c}_{\tau}\right\|^{2}+\lambda} \\
\operatorname{MMSE}\left(d, \tau, N_{f}=\infty\right) & =\frac{1}{2 \pi} \int_{-\pi}^{\pi} \frac{\lambda}{|C(\omega, \tau)|^{2}+\lambda} d \omega
\end{aligned}
$$

where $\lambda$ is the noise-to-signal ratio defined by

$$
\lambda=\frac{E\left\{w^{2}[k]\right\}}{E\left\{s^{2}[k]\right\}} .
$$

A detailed derivation of (10) and (11) can be found in [9].

Now, consider the timing offset for a one-tap linear equalizer. Since the delay term $d$ appears only in $c_{\tau}[d]$ in $\operatorname{MMSE}\left(d, \tau, N_{f}=1\right)$, the attainable minimum MMSE with respect to the $d$ is given by $\min _{d} \operatorname{MMSE}\left(d, \tau, N_{f}=1\right)$, and hence, the optimal timing offset that minimizes the MMSE for the one-tap equalizer is given by

$$
\tau_{\text {opt }}=\max _{\tau} \frac{\left\|\mathbf{c}_{\tau}\right\|_{\infty}^{2}}{\left\|\mathrm{c}_{\tau}\right\|^{2}+\lambda}
$$

where $\|\cdot\|_{\infty}$ denotes the $\ell_{\infty}$ norm.

The MMSE-timing function (13) provides a geometrical interpretation of the timing offset optimization for a one-tap equalizer. Consider normalized $\mathrm{c}_{\tau}$,

$$
\overline{\mathrm{c}}_{\tau}=\frac{\mathrm{c}_{\tau}}{\left\|\mathrm{c}_{\tau}\right\|} .
$$

$\overline{\mathrm{c}}_{\tau}$ lies on an $N_{c}$-dimensional sphere as $\tau$ varies from $-1 / 2$ to $1 / 2$, and $\tau_{\text {opt }}$ is chosen so that (13) is as large as possible, which occurs when $\left\|c_{\tau}\right\|_{\infty}^{2}$ is maximized. Thus, the effect of using the optimal timing offset for a one parameter equalizer can be seen directly by looking at the taps of $c_{\tau}$. Intersymbol interference (ISI) is minimized by maximizing the magnitude of the tap corresponding to the system delay and, at the same time, suppressing all others.

On the other hand, for the timing offset optimized for infinite length equalizers, in [4], the following approximation of (11) is proposed:

$$
\operatorname{MMSE}\left(\tau, N_{f}=\infty\right) \approx \frac{1}{\left\|\mathrm{c}_{\tau}\right\|^{2}+\lambda}
$$

In this case, minimizing MMSE is equivalent to maximizing the channel power $\left\|c_{\tau}\right\|^{2}$ and, consequently, improving the signal (including ISI components) to noise ratio, since an infinite length equalizer can perfectly restore signals from ISI.

Apparently, increasing total signal power does not necessarily improve the equalizer output MSE, especially when the multipath is severe and the equalizer is not long enough to mitigate the multipath. The following example confirms that applying the timing offset optimized for an infinite length equalizer to a finite equalizer (15) performs worse than the timing offset optimized for the one-tap equalizer (13).

Example 1: Consider a multipath channel

$$
m(t)=\delta(t)+0.6 \delta(t-1.4 T)-0.3 \delta(t-12.6 T)
$$

using a raised-cosine pulse-shaping filter with a roll-off factor $\alpha=0.5$ under SNR $=30 \mathrm{~dB}$. Numerical calculation shows that the optimal timing offsets for $N_{f}=1, \tau_{N_{f}=1}$ and the optimal timing offset for $N_{f}=\infty, \tau_{N_{f}=\infty}$ are given by

$$
\tau_{N_{f}=1}=0.07, \quad \tau_{N_{f}=\infty}=0.83 .
$$

For these two timing offsets, Fig. 2 plots the MSE of a) MMSE linear of equalizers of all lengths from 1 to 25 and $b$ ) the MMSE decision feedback equalizer (DFE) with feedback filter of fixed length 12 and feedforward equalizers with all lengths from 1 to 25 . The optimal timing offset for each finite equalizer length has been calculated numerically, and its MMSE equalizer MSE is depicted as a dotted line. In all cases, when the timing is optimized for a one-tap equalizer, the performance is better than when it is optimized for an infinite-length equalizer.

This example does not represent a peculiar case. In fact, one can confirm this trend for a set of randomly generated channels, as reported in [10]. The next section presents an adaptive scheme approximating the timing offset for the one-tap equalizer without help of known training sequence.

\section{TIMING OfFSET BASED ON DISPERSION MiNIMIZATION}

\section{A. DM-Timing Offsets}

Instead of directly minimizing the MSE, which requires training signal or analog channel estimation, [7] has proposed minimizing the mean dispersion of the sampler output signal, i.e.,

$$
J(\tau)=E\left\{\left(\left|r_{k}(\tau)\right|^{2}-\gamma\right)^{2}\right\}
$$

where $\gamma$ is a constant corresponding to the source statistics. $(\gamma=$ $E|s|^{4} / E|s|^{2}$; for details, see [11].) The cost function is known as the constant modulus (CM) cost and is widely used for blind equalization [11]. In order to approximate $\operatorname{MMSE}\left(\tau, N_{f}=1\right)$ 


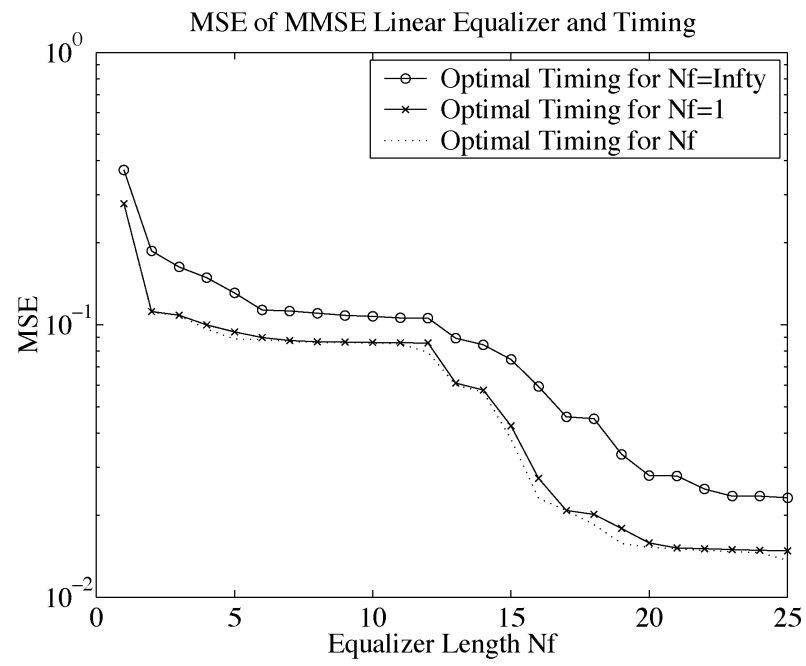

a)

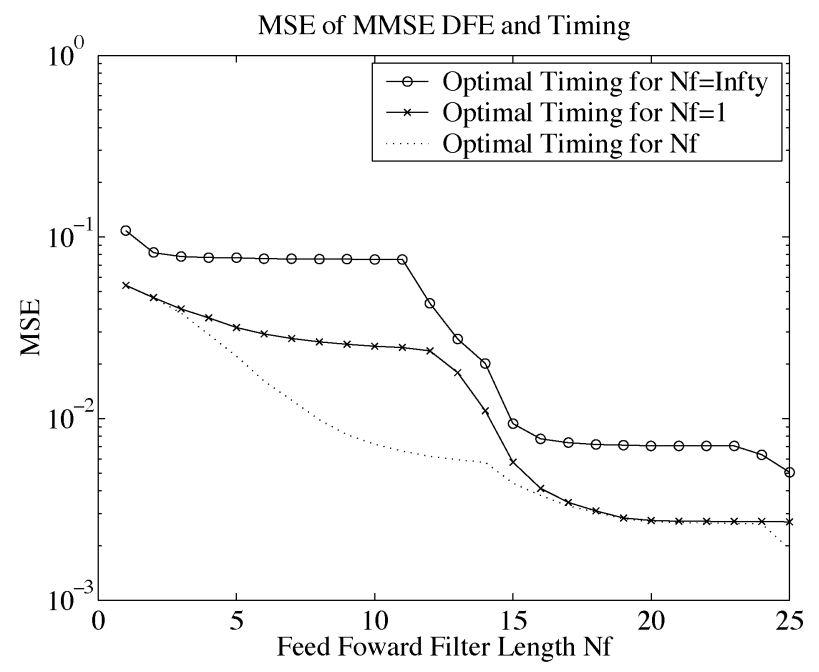

b)

Fig. 2. MMSE equalizer output MSE and Timing offset. (a) Linear equalizer. (b) DFE with feed back filter length 12.

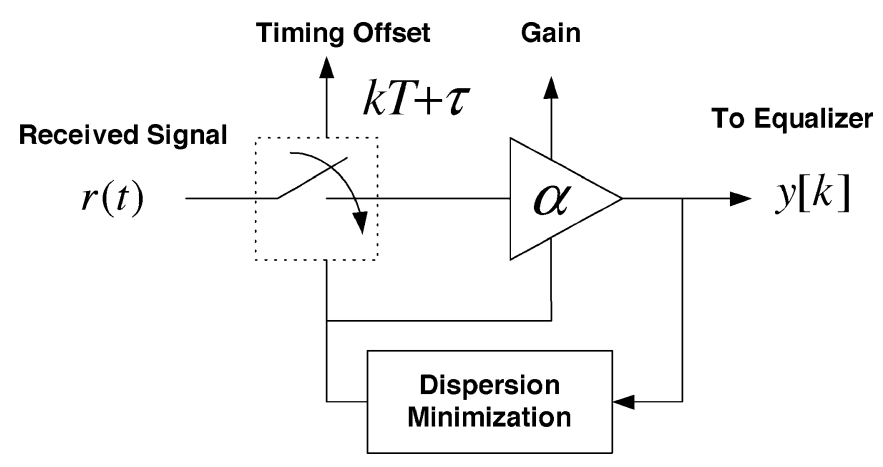

Fig. 3. Dispersion minimization timing offset.

with respect to a one-tap equalizer $\alpha$, we proposed to rewrite the cost function (18) as

$$
J(\tau, \alpha)=E\left\{\left(\left|\alpha r_{k}(\tau)\right|^{2}-\gamma\right)^{2}\right\}
$$

as illustrated in Fig. 3. Using a stochastic gradient algorithm [12] to minimize (19) by recursive selection of $\tau_{k}$ and $\alpha_{k}$ gives the dispersion minimization (DM) timing algorithm

$$
\begin{aligned}
\tau_{k+1} & =\tau_{k}-\mu\left(\left|\alpha_{k} r_{k}\left(\tau_{k}\right)\right|^{2}-\gamma\right)\left|\alpha_{k}\right|^{2} r_{k}\left(\tau_{k}\right) \frac{\partial r_{k}\left(\tau_{k}\right)}{\partial \tau_{k}} \\
\alpha_{k+1} & =\alpha_{k}-\mu\left(\left|r_{k}\left(\tau_{k}\right)\right|^{2}-\gamma\right) \alpha_{k}\left|r_{k}\left(\tau_{k}\right)\right|^{2}
\end{aligned}
$$

where $\partial r_{k}\left(\tau_{k}\right) / \partial \tau_{k}$ can be obtained numerically when a closed form is unavailable.

Expansion of the DM-cost function (19) provides detailed information on the DM timing offsets. Setting $\lambda=\sigma_{w}^{2} / \sigma_{s}^{2}$ and assuming $\sigma_{s}^{2}=1$, the DM cost function (18) is given by the following (for the derivations, see Appendix A):

$$
\begin{aligned}
J(\tau, \alpha)=\alpha^{4}\left(\eta_{s}\left\|\mathrm{c}_{\tau}\right\|_{4}^{4}+\kappa_{w}\right. & \left.\left(\left\|\mathrm{c}_{\tau}\right\|^{2}+\lambda\right)^{2}\right) \\
& -2 \alpha^{2}\left(\kappa_{s}\left\|\mathrm{c}_{\tau}\right\|^{2}+\lambda\right)+\kappa_{s}^{2}
\end{aligned}
$$

where $\kappa_{s}$ and $\kappa_{w}$ are called kurtosis of the source $s$ and the noise $w$, respectively, and are defined by

$$
\begin{aligned}
& \kappa_{s}=\frac{E\left\{|s|^{4}\right\}}{E\left\{|s|^{2}\right\}^{2}}, \quad \kappa_{w}=\frac{E\left\{|w|^{4}\right\}}{E\left\{|w|^{2}\right\}^{2}} \\
& \eta_{s}=\kappa_{w}-\kappa_{s}
\end{aligned}
$$

where $\|\cdot\|_{4}$ denotes the $\ell_{4}$ norm. Note that $\kappa_{w}=3$ for a real Gaussian $\{w\}$ and $\kappa_{w}=2$ for a complex Gaussian $\{w\}$. Since the cost function (22) is a quadratic function of $\alpha$, there exists a unique optimum $\alpha$ for each $\tau$. Using this fact, (22) can be written as a function of only $\tau$, which leads to the following result.

Theorem 1 (DM-Minima): The DM-timing offset for a single sensor is given by

$$
\tau_{\mathrm{DM}}=\arg \max _{\tau} \frac{\left\|\mathrm{c}_{\tau}\right\|_{4}^{2}}{\left\|\mathrm{c}_{\tau}\right\|^{2}+\lambda} .
$$

Proof: See Appendix B.

There is a similarity between MMSE-timing and DM-timing. Recall the notation $\overline{\mathrm{c}}_{\tau}=\mathrm{c}_{\tau} /\left\|\mathrm{c}_{\tau}\right\|$. In the absence of noise, the optimal MMSE timing offset for a one-tap equalizer is given by $\arg \max _{\tau}\left\|\overline{\mathrm{c}}_{\tau}\right\|_{\infty}$, whereas the optimal DM-timing offset is $\arg \max _{\tau}\left\|\overline{\mathrm{c}}_{\tau}\right\|_{4}$. The only difference between these two timing offsets is the norm used $\left(\ell_{4}\right.$ or $\left.\ell_{\infty}\right)$. In both cases, with $\tau$ constrained to $-1 / 2<\tau<1 / 2$, the optimal $\overline{\mathrm{c}}_{\tau}$ lies on the unit $\left(\ell_{4}\right.$ or $\ell_{\infty}$ ) sphere.

\section{B. Unimodality Condition of DM-Timing}

This section establishes a concrete set of conditions under that the DM cost function bears a unique maximum, which seems to be satisfied in most practical situations. The immediate implication of this result is that the algorithm's asymptotic behavior is insensitive to the initial conditions. Call

$$
I(\tau)=\frac{\left\|\mathrm{c}_{\tau}\right\|_{4}^{2}}{\left\|\mathrm{c}_{\tau}\right\|^{2}+\lambda}
$$


TABLE I

DM-TIMING FunCTION COEFFICIENTS VERSUS ROLL-OFF FACTOR OF RAISED COSINE

\begin{tabular}{c|c|c|c|c}
\hline Roll-off Factor & $\alpha=0.1$ & $\alpha=0.3$ & $\alpha=0.5$ & $\alpha=1$ \\
\hline$H_{2}(0)$ & 0.9749 & 0.9249 & 0.8749 & 0.7499 \\
$\left|H_{2}(1)\right|$ & 0.0124 & 0.0374 & 0.0624 & 0.1249 \\
$H_{4}(0)$ & 0.6647 & 0.6512 & 0.6272 & 0.5411 \\
$\left|H_{4}(1)\right|$ & 0.1675 & 0.1739 & 0.1849 & 0.2186 \\
$\left|H_{4}(2)\right|$ & $1 \times 10^{-5}$ & $2 \times 10^{-4}$ & 0.0013 & 0.0106 \\
$\left|H_{4}(3)\right|$ & $\doteq 0$ & $\doteq 0$ & $\doteq 0$ & $1 \times 10^{-5}$ \\
$H_{4} G_{2}-2 H_{2} G_{4}-5 H_{2} H_{4}$ & 0.2520 & 0.0945 & -0.0633 & -0.4876 \\
\hline
\end{tabular}

the DM-timing cost. The following analysis focuses on the unimodality of $I(\tau)$. Let $H(f)$ denote the Fourier transform of $c(t)$, which is the analog channel, and denote

$$
H_{n}(f)=\underbrace{H(f) \star \cdots \star H(f)}_{n \text { times }}
$$

where $\star$ indicates convolution operator. From the Fourier series expansion of $\left\|c_{\tau}\right\|^{2}$ and $\left\|c_{\tau}\right\|_{4}^{4}$

$$
\begin{aligned}
& \left\|\mathrm{c}_{\tau}\right\|^{2}=\sum_{k=-\infty}^{\infty} c^{2}(t+k T)=\frac{1}{T} \sum_{k=-\infty}^{\infty} H_{2}\left(\frac{k}{T_{s}}\right) e^{j 2 \pi \frac{k t}{T_{s}}} \\
& \left\|\mathrm{c}_{\tau}\right\|_{4}^{4}=\sum_{k=-\infty}^{\infty} c^{4}(t+k T)=\frac{1}{T} \sum_{k=-\infty}^{\infty} H_{4}\left(\frac{k}{T_{s}}\right) e^{j 2 \pi \frac{k t}{T_{s}}} .
\end{aligned}
$$

Assuming $T=1$, since $c(t)$ is bandlimited, we have

$$
H_{n}(f)=0 \text { for }|f|>\frac{n}{T}, \quad \text { for } \quad n=1,2,4 \text {. }
$$

From (28) and (29)

$$
\begin{aligned}
\left\|\mathrm{c}_{\tau}\right\|^{2} & =H_{2}(0)+2\left|H_{2}(1)\right| \cos \left(2 \pi \tau+\angle H_{2}(1)\right) \\
\left\|\mathrm{c}_{\tau}\right\|_{4}^{4} & =H_{4}(0)+2 \sum_{m=1}^{3}\left|H_{4}(m)\right| \cos \left(2 m \pi \tau+\angle H_{4}(m)\right) .
\end{aligned}
$$

The values of $\left\{H_{2}(k), H_{4}(m)\right\}$ are determined by the multipath channel $m(t)$ and the pulse-shaping filter $p(t)$. In practice, a commonly used pulse-shaping filter is a raised cosine function with spectrum

$P(f)= \begin{cases}T, & 0 \leq|f| \leq \frac{(1-\alpha)}{2 T} \\ \frac{T}{2}\left[1+\cos \left(\frac{\pi T}{\alpha}\left(|f|-\frac{1-\alpha}{2 T}\right)\right],\right. & \frac{1-\alpha}{2 T} \leq|f| \leq \frac{1+\alpha}{2 T} \\ 0, & |f|>\frac{1+\alpha}{2 T}\end{cases}$

where $\alpha$ is the roll-off factor determining the excess bandwidth. A roll-off factor from 0.1 to 0.3 is commonly used in practice (for example, [13] and [14]).

For a multipath $m(t)=\sum_{k=0}^{N_{c}-1} \rho_{k} \delta\left(t-\tau_{k}\right), H(f)$ is given by

$$
H(f)=P(f) \sum_{k=0}^{N_{c}-1} \rho_{k} e^{-2 \pi j \tau_{k} f}
$$

Since $|h(f) \star g(f)| \leq|h(f)| \star|g(f)|$ for given complex functions $h(f)$ and $g(f)$, we have

$$
\begin{aligned}
\left|H_{n}(f)\right| \leq P_{n}(f) & \left(\sum_{k=0}^{N_{c}-1}\left|\rho_{k}\right|\right)^{n} \\
& \text { where } P_{n}(f)=\underbrace{P(f) \star \cdots \star P}_{n \text { times }}(f) .
\end{aligned}
$$

(For the detailed derivation, see Appendix E.) For a roll-off factor from 0.1 to 0.3 , the tails of $P(f)$ are quite small, and consequently, the values $\left|H_{4}(2)\right|,\left|H_{4}(3)\right|$, and $\left|H_{4}(4)\right|$ are vanishingly small in comparison with other values such as $\left|H_{4}(0)\right|$ and $\left|H_{4}(1)\right|$ (see Table I). Thus, a good approximation of $\left\|\mathbf{c}_{\tau}\right\|_{4}^{4}$ is

$$
\left\|\mathrm{c}_{\tau}\right\|_{4}^{4} \approx H_{4}(0)+2\left|H_{4}(1)\right| \cos \left(2 \pi \tau+\angle H_{4}(1)\right)
$$

which simplifies the DM-timing function to

$$
I(\tau)=\frac{\sqrt{H_{4}(0)+2\left|H_{4}(1)\right| \cos \left(2 \pi \tau+\angle H_{4}(1)\right)}}{H_{2}(0)+2\left|H_{2}(1)\right| \cos \left(2 \pi \tau+\angle H_{2}(1)\right)+\lambda} .
$$

For the rest of this paper, we use the approximation (36) for further investigation in order to avoid extremely difficult computational complexity due to the high-frequency term in (32).

Theorem 2 (Unimodality): Denote

$$
\begin{aligned}
& G_{2}=H_{2}(0)+\lambda, \quad G_{4}=H_{4}(0), \\
& H_{2}=2\left|H_{2}(1)\right|, \quad H_{4}=2\left|H_{4}(1)\right| .
\end{aligned}
$$

Then

$$
I(\tau)=\frac{\sqrt{G_{4}+H_{4} \cos \left(2 \pi \tau+\angle H_{4}(1)\right)}}{G_{2}+H_{2} \cos \left(2 \pi \tau+\angle H_{2}(1)\right)}
$$

is unimodal, i.e. has a unique maximum, when the following condition holds:

$$
H_{4} G_{2}-2 H_{2} G_{4}>5 H_{2} H_{4}
$$

Proof: See Appendix C.

Notice that the condition (40) in Theorem 2 is satisfied when the tails $H_{2}(1)$ and $H_{4}(1)$ decrease rapidly. Table I shows that for small roll-off factors $\alpha \leq 0.3$, (40) is satisfied, which implies that the DM-algorithm has only one minimum. This holds for any multipath channel. In fact, (40), which is given in Theorem 2 , is a loose bound. A tighter bound can be found under slightly more complicated conditions, as shown in [10]. 


\section{Performance of DM-Timing}

The MSE performance of the dispersion minimization approach has been intensively studied for the equalization applications under the assumption that perfect equalization is possible [15], [16]. It has been shown that the dispersion minimization approach can achieve near-MMSE performance. However, this result cannot be applied to the timing offset problem here, since the channel vectors parameterized by timing offsets are constrained. In fact, the geometrical arguments used to show the near-optimal MSE performance of dispersion minimization [17], [18] fail for the constrained cases [10]. On the other hand, due to the nonlinearity of the cost functions for DM-timing and MMSE-timing, it is a virtually impossible task to establish a rigorous MSE bound for a general channel. Therefore, the MSE performance of DM-timing is investigated by comparing the location of the three different timing offsets, DM-timing, MMSE-timing for a one-tap equalizer, and MMSE-timing offset for an infinite length equalizer (the existing blind timing offset method). This comparison demonstrates the relative MSE performance of the three timing methods when used with short equalizers and provides intuition about the different approaches.

Consider a multipath channel $c(t)$ of $N_{c}$ rays with a raisedcosine pulse shape and its Fourier transformation, as given in (33) and (34)

$c(t)=\sum_{k=0}^{N_{c}-1} \rho_{k} \delta\left(t-\tau_{k}\right) \star p(t), \quad H(f)=\sum_{k=0}^{N_{c}-1} \rho_{k} e^{-2 j \pi \tau_{k} f} P(f)$.

Recall that $H_{2}(f):=H(f) \star H(f)$ and that $H_{2}:=2\left|H_{2}(1)\right|$, $H_{4}:=2\left|H_{4}(1)\right|, G_{2}:=H_{2}(0)+\lambda$, and $G_{4}:=H_{4}(1)$. Let

$$
\phi_{2}:=\angle H_{2}(1), \quad \phi_{4}:=\angle H_{4}(1) .
$$

The MMSE timing offset for infinite length MMSE equalizer, which is denoted by $\tau_{M \infty}$, is given from (15)

$$
\tau_{M \infty}=\arg \min \frac{1}{H_{2} \cos \left(2 \pi \tau+\phi_{2}\right)+G_{2}}=-\frac{1}{2 \pi} \phi_{2} .
$$

On the other hand, the MMSE timing offset for the one-tap MMSE equalizer, which is denoted by $\tau_{M 1}$, is given by

$$
\begin{aligned}
\tau_{M 1} & =\arg \max \frac{\|c(\tau)\|_{\infty}^{2}}{H_{2} \cos \left(2 \pi \tau+\phi_{2}\right)+G_{2}} \\
\tau_{\mathrm{DM}} & =\arg \max \frac{H_{4} \cos \left(2 \pi \tau+\phi_{4}\right)+G_{4}}{\left(H_{2} \cos \left(2 \pi \tau+\phi_{2}\right)+G_{2}\right)^{2}} .
\end{aligned}
$$

These formulas imply that $\tau_{M 1}$ is shifted from $\arg \max \|\mathrm{c}(\tau)\|$ toward $\tau_{M \infty}+0.5$, and $\tau_{\mathrm{DM}}$ is shifted from $-\left(\phi_{4} / 2 \pi\right)$ toward $\tau_{M \infty}+0.5$. For example, $\tau_{\mathrm{DM}}$ satisfies (45), shown at the bottom of the page. Notice that when $\phi_{2}=\phi_{4}, \tau_{\mathrm{DM}}=$ $-\left(\phi_{4} / 2 \pi\right)$, and consequently, $\tau_{\mathrm{DM}}=\tau_{M \infty}$. Depending on the channel, $\tau_{\mathrm{DM}}, \tau_{M 1}$, and $\tau_{M \infty}$ agree or disagree each other. In general, for mild multipath channels, $\|c(\tau)\|_{4}^{2}$ is a reasonable approximation of $\|c(\tau)\|_{\infty}^{2}$, and thus, $\tau_{D} M$ is close to $\tau_{M 1}$, whereas $\tau_{M \infty}$ usually deviates from $\tau_{M 1}$. As the severity of the channel increases, $\tau_{\mathrm{DM}}$ tends to locate away from $\tau_{M 1}$ and close to $\tau_{M \infty}$, and consequently, its MSE performance is degraded. In the next subsections, we will further investigate these MSE properties of DM-timing for the cases where $\|c(\tau)\|_{\infty}^{2}$ can be approximated by a manageable function. To proceed further, we need the following Lemma.

Lemma 1: Let $\tau_{0}$ and $\tau_{1}$ denote delays in the time domain. Then, we have

$$
\begin{gathered}
\text { i) } \angle\left(e^{-j 2 \pi \tau_{0} f} P(f) \star e^{-j 2 \pi \tau_{1} f} P(f)\right) \\
=-2 \pi f \frac{\tau_{0}+\tau_{1}}{2}, \\
\text { ii) }\left|e^{-j 2 \pi \tau_{0} f} P(f) \star e^{-j 2 \pi \tau_{1} f} P(f)\right| \\
\rightarrow P_{2}(f) \text { as }\left|\tau_{0}-\tau_{1}\right| \rightarrow 0 \\
\text { iii) }\left|e^{-j 2 \pi \tau_{0} f} P(f) \star e^{-j 2 \pi \tau_{1} f} P(f)\right| \\
\rightarrow 0 \text { as }\left|\tau_{0}-\tau_{1}\right| \rightarrow \infty .
\end{gathered}
$$

Proof: See Appendix D.

\section{A. Case 1: Pure Delay}

First, as a simple example, consider a pure delay channel, i.e., $c(t)=p\left(t-\tau_{0}\right)$. Since $c(t)$ is a raised cosine delayed by $\tau_{0}$ and sampled at $\tau_{0}$, the one-tap MMSE equalizer as well as the infinite length equalizer has the minimum MSE. On the other hand

$$
H_{n}(f)=e^{-j 2 \pi \tau_{0} f} P_{n}(f) .
$$

Since $P_{n}$ is real valued, $\phi_{2}=\phi_{4}=-2 \pi \tau_{0}$. Thus, from (45), we have $\tau_{\mathrm{DM}}=\tau_{M \infty}=\tau_{0}$. In this pure delay case, $\tau_{\mathrm{DM}}, \tau_{M 1}$, and $\tau_{M \infty}$ agree with each other.

\section{B. Case 2: Clustered Channels}

Assume that the multipath channel $c(t)$ given by

$$
c(t)=\sum_{i=0}^{N-1} \rho_{i} p\left(t-\tau_{i}\right)
$$

is clustered in the sense that $\max \left\{\mid \tau_{i}-\tau_{j} \| 0 \leq i, j<N\right\}<1$. Since the delays are clustered, the peaks for each delay merge into a single peak. This can be shown by the following approximation in the region $\left|t-\tau_{i}\right|<1$ :

$$
p(t)=\operatorname{sinc}(t) \frac{\cos (\pi \alpha t)}{1-4 \alpha^{2} t^{2}} \approx \operatorname{sinc}(t) \approx \cos \left(\frac{\pi t}{2}\right)
$$

where we have used

$$
\begin{aligned}
\operatorname{sinc}(t) & \approx 1-\frac{\pi^{2} t^{2}}{6}+\frac{\pi^{4} t^{4}}{5 !}-\cdots \\
& \approx 1-\frac{\pi^{2} t^{2}}{8}+\frac{\pi^{4} t^{4}}{2^{4} 4 !}-\cdots \\
& =\cos \left(\frac{\pi t}{2}\right) .
\end{aligned}
$$

$$
\tau_{\mathrm{DM}}=-\frac{\phi_{4}}{2 \pi}-\frac{1}{2 \pi} \tan ^{-1} \frac{2 H_{2} \sin \left(\phi_{2}-\phi_{4}\right)\left(G_{4}+H_{4} \cos \left(2 \pi \tau_{\mathrm{DM}}+\phi_{4}\right)\right)}{G_{2} H_{4}-2 H_{2} G_{4} \cos \left(\phi_{2}-\phi_{4}\right)-H_{2} H_{4} \cos \left(2 \pi \tau_{\mathrm{DM}}-\phi_{2}\right)}
$$


Therefore

$c(t) \approx \sum_{i=0}^{N-1} \rho_{i} \cos \left(\pi \frac{t-\tau_{i}}{2}\right)=A \cos \left(\pi \frac{t}{2}+\tau_{A}\right)$, for $\left|t-\tau_{i}\right|<1$

for a magnitude $A$ and phase $\tau_{A}$. From $\sum_{i=0}^{N-1} \rho_{i} \cos (\pi(t-$ $\left.\left.\tau_{i} / 2\right)\right)=\Re\left(\sum_{i=0}^{N-1} \rho_{i} e^{j \pi\left(t-\tau_{i} / 2\right)}\right), A$ and $\tau_{A}$ are given by

$$
A=\left|\sum_{i=0}^{N-1} \rho_{i} e^{j \pi \frac{-\tau_{i}}{2}}\right|, \quad \tau_{A}=L \sum_{i=0}^{N-1} \rho_{i} e^{j \pi \frac{-\tau_{i}}{2}} .
$$

Thus, we have

$$
\arg \max \|c(\tau)\|_{\infty}^{2}=-\frac{2 \tau_{A}}{\pi}
$$

$\left|t-\tau_{i}\right| \ll 1$ for all $i$.

On the other hand

$$
\begin{aligned}
H_{2}(f)= & \sum_{i=0}^{N-1} \rho_{i}^{2} e^{-j 2 \pi \tau_{i} f} P_{2}(f) \\
& +\sum_{i \neq j} e^{-j 2 \pi \tau_{i} f} P(f) \star e^{-j 2 \pi \tau_{j} f} P(f) \\
\approx & \sum_{i=0}^{N-1} \rho_{i}^{2} e^{-j 2 \pi \tau_{i} f} P_{2}(f) \\
& +\sum_{i \neq j} \rho_{i} \rho_{j} e^{-j 2 \pi f \frac{\tau_{i}+\tau_{j}}{2}} P_{2}(f) \\
= & \left(\sum_{i=0}^{N-1} \rho_{i} e^{-j \pi \tau_{i} f}\right)^{2} P_{2}(f)
\end{aligned}
$$

where, in (57), we have used property ii) of Lemma 1 , and the assumption that the multipath channel is clustered. Similarly, since $P_{2}$ is again real and symmetric, Lemma 1 shows that

$$
H_{4}(f) \approx\left(\sum_{i=0}^{N-1} \rho_{i} e^{-j \frac{\pi}{2} \tau_{i} f}\right)^{4} P_{4}(f)
$$

and consequently

$$
\begin{aligned}
& \phi_{2} \approx 2 \angle\left(\sum_{i=0}^{N-1} \rho_{i} e^{-j \pi \tau_{i}}\right)=4 \tau_{A} \\
& \phi_{4} \approx 4 \angle\left(\sum_{i=0}^{N-1} \rho_{i} e^{-j \frac{\pi}{2} \tau_{i}}\right)=4 \tau_{A} .
\end{aligned}
$$

Therefore, we have

$$
\arg \max \left\|\mathrm{c}_{\tau}\right\|_{4}^{4} \approx-\frac{2}{\pi} \tau_{A} \approx \arg \max \left\|\mathrm{c}_{\tau}\right\|_{\infty}^{4} .
$$

This implies that $\tau_{M \infty}$ and $\tau_{\mathrm{DM}}$ are shifted from the same location toward $\tau_{M \infty}+0.5$, and thus, they are located close to each other (close to MSE performance), whereas $\tau_{M \infty}$ may not be located close to $\tau_{M 1}$ (different MSE performance), as the simulation results in Section IV-D confirms.

\section{Case 3: Sparse Channels}

Now assume that the multipath channel $c(t)=\sum_{i} \rho_{i} p\left(t-\tau_{i}\right)$ is sparse in the sense that

$$
\left|\tau_{i}-\tau_{j}\right| \gg 1, \text { for all } i \neq j \text {. }
$$

Without loss of generality, $\rho_{0}$ is assumed to be the maximum multipath magnitude, and $\rho_{0}=1$. Since the multipath is sparse, near the highest peak $t=\tau_{0}$, the contribution from the tails of other peaks can be ignored, i.e., locally at $t=\tau_{0}$

$$
\mathrm{c}(t) \approx p\left(t-\tau_{0}\right)
$$

Thus

$$
\arg \max \left\|\mathrm{c}_{\tau}\right\|_{\infty}^{4} \approx \tau_{0} .
$$

On the other hand

$$
\begin{aligned}
H_{2}(f)= & \sum_{i=0}^{N-1} \rho_{i}^{2} e^{-j 2 \pi \tau_{i} f} P_{2}(f) \\
& +\sum_{i \neq j} e^{-j 2 \pi \tau_{i} f} P(f) \star e^{-j 2 \pi \tau_{j} f} P(f) \\
\approx & \sum_{i=0}^{N-1} \rho_{i}^{2} e^{-j 2 \pi \tau_{i} f} P_{2}(f)
\end{aligned}
$$

where we have used property iii) of Lemma 1 and the assumption of channel sparsity. Similarly

$$
H_{4}(f) \approx \sum_{i=0}^{N-1} \rho_{i}^{4} e^{-j 2 \pi \tau_{i} f} P_{4}(f)
$$

and consequently

$$
\begin{aligned}
& \phi_{2} \approx L \sum_{i=0}^{N-1} \rho_{i}^{2} e^{-j 2 \pi \tau_{i}} \\
& \phi_{4} \approx L \sum_{i=0}^{N-1} \rho_{i}^{4} e^{-j 2 \pi \tau_{i}} .
\end{aligned}
$$

Notice that $\phi_{2}$ and $\phi_{4}$ are the nonlinear average of $\tau_{i}$ 's weighted by the power of the magnitude of multipath. $\phi_{2}=\phi_{4}$ when $\rho_{i}=0$ for $i>0$ (pure delay) and $\rho_{i}=1$ for all $i$ (the worst multipath). For the other cases, $\phi_{4}$ is closer to $-2 \pi \tau_{0}$ than $\phi_{2}$ since $\rho^{4}$ is smaller than $\rho^{2}$. For example, in a two-tap channel case, assuming $\tau_{0}=0, \phi_{2}$ and $\phi_{4}$ are given by

$$
\phi_{k}=-\tan ^{-1} \frac{\rho_{1}^{k} \sin \left(2 \pi \tau_{1}\right)}{1+\rho_{1}^{k} \cos \left(2 \pi \tau_{1}\right)}, \quad k=2,4
$$

and for $\tau_{1}=0.25 \bmod 1$, this is simply $\phi_{k}=-\tan ^{-1}\left(\rho_{1}^{k}\right)$ $(k=2,4)$. Fig. 4(a) plots three different timing offset for $\tau_{1}=10.25$ as $\rho_{1}$ increases 0 to $1 . \tau_{M \infty}$ follows the trajectory of $(1 / 2 \pi) \tan ^{-1}\left(\rho_{1}\right)$ from 0 to $1 / 8\left((1 / 2 \pi) \tan ^{-1}(1)\right)$, and $\tau_{M 1}$ is shifted toward $\tau_{M \infty}+0.5$ (or, equivalently, $\left.\tau_{M \infty}-0.5\right)$ from $\tau_{0}=0 . \tau_{\mathrm{DM}}$ is located close to $\tau_{M 1}$ until $\rho_{1}=0.5$ and rapidly approaches $\tau_{M \infty}$ as the multipath becomes more severe. The corresponding MSE performance is shown in Fig. 4(b). Notice that the MSE exhibits the same pattern for timing offset locations in Fig. 4(a).

This two-tap example suggests that under mild channels the DM-timing offset yields near MMSE performance for the one-tap MMSE equalizer, and under severe multipath distortion, the DM-timing offset achieves better MSE performance than the conventional OEM algorithm. The simulation results in the following subsection experimentally confirms this MSE performance of DM-timing for general channels.

\section{DM-Timing Offset MSE Performance Simulation}

This subsection presents simulation results comparing MSE performance of different timing offset $\tau_{\mathrm{DM}}, \tau_{M 1}$, and $\tau_{M \infty}$ 


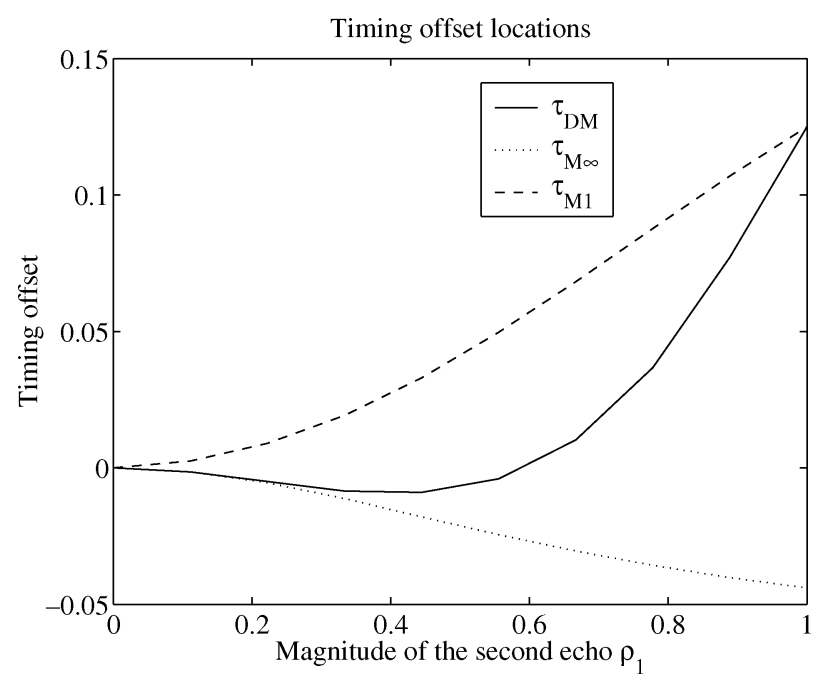

a)

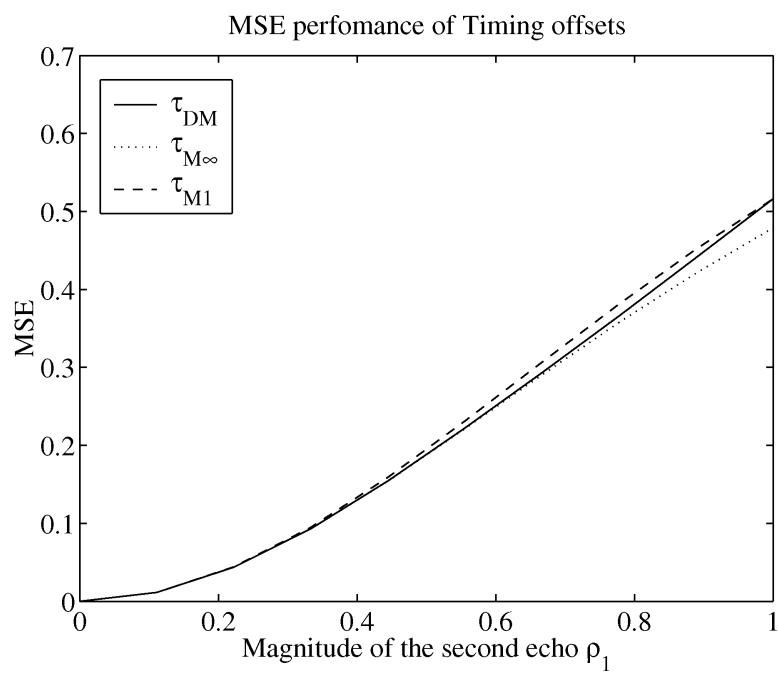

b)

Fig. 4. DM-timing and MSE-timing for two-tap channels. (a) Timing offset locations. (b) MSE performance of timing offsets.

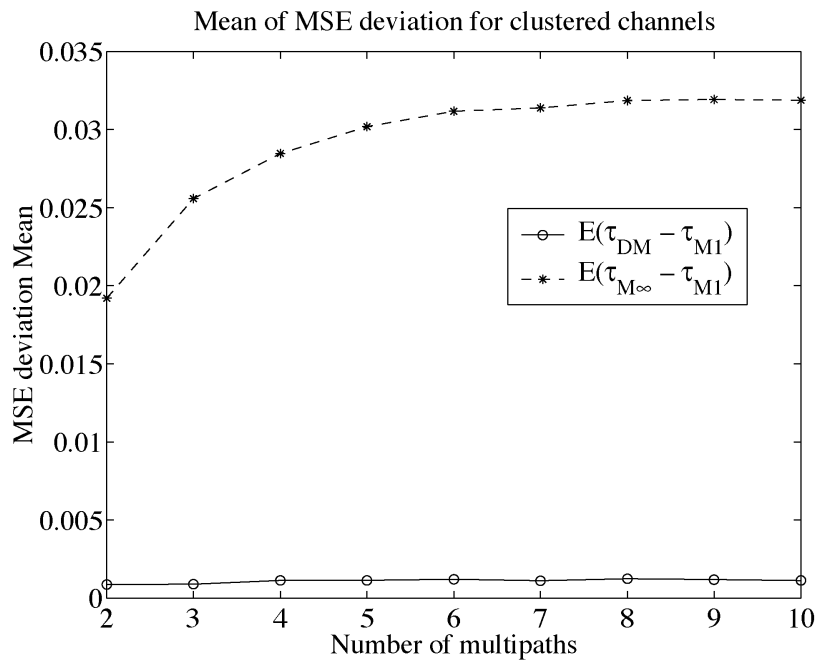

a)

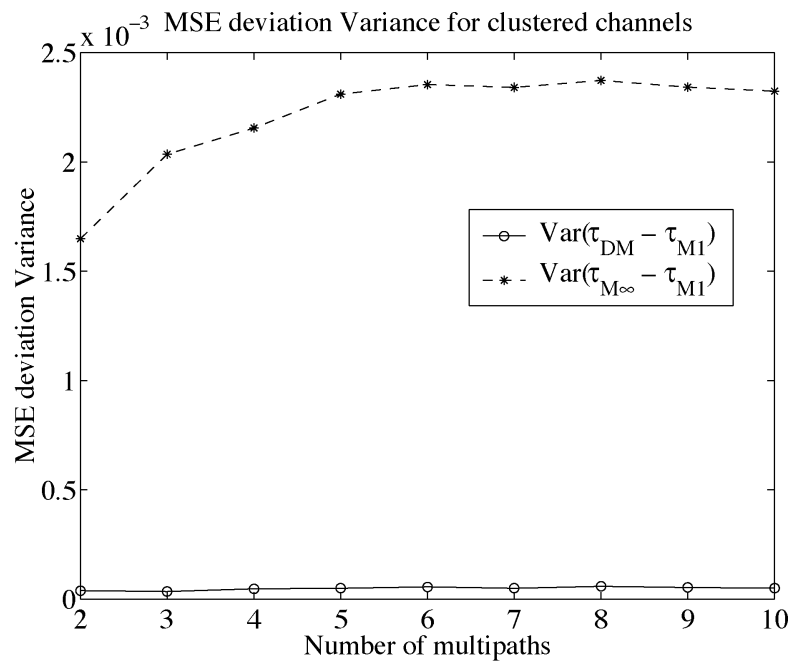

b)

Fig. 5. DM-timing and MSE-timing for clustered channels. a) MSE deviation mean; b) MSE deviation variance.

for a one-tap equalizer under $30-\mathrm{dB}$ noise. For the number of multipath rays from 2 to 10,10000 different randomly generated multipath channels are used to compute mean and variance of MSE difference, i.e., $E\left(\operatorname{MSE}\left(\tau_{\mathrm{DM}}\right)-\operatorname{MSE}\left(\tau_{M 1}\right)\right)$, $E\left(\operatorname{MSE}\left(\tau_{M \infty}\right)-\operatorname{MSE}\left(\tau_{M 1}\right)\right), \operatorname{Var}\left(\operatorname{MSE}\left(\tau_{\mathrm{DM}}\right)-\operatorname{MSE}\left(\tau_{M 1}\right)\right)$, and $\operatorname{Var}\left(\operatorname{MSE}\left(\tau_{M \infty}\right)-\operatorname{MSE}\left(\tau_{M 1}\right)\right)$. For the clustered scenario, the delays are confined to $\tau_{k} \in[0,1]$. For the mild channel scenario, the magnitude of the cursor tap is set to 1 , and the rest are confined to below 0.5 (less than $3 \mathrm{~dB}$ multipath), and for severe multipath, the rest are set to above 0.5 . The simulation results shows the MSE performance of the DM-timing offset predicted in the previous subsections.

For clustered channels, mean and variance of the MSE difference between $\tau_{\mathrm{DM}}$ and $\tau_{M 1}$ are significantly lower than that between $\tau_{M \infty}$ and $\tau_{M 1}$. This indicates that $\tau_{\mathrm{DM}}$ is a good approximation for $\tau_{M 1}$, whereas $\tau_{M \infty}$ may not be for certain channels.

For the nonclustered channels, $\tau_{\mathrm{DM}}$ outperforms $\tau_{M \infty}$. For mild channels, the mean and variance are small, which indicates that $\tau_{\mathrm{DM}}$ is still a good approximation for $\tau_{M \infty}$. Since the magnitude of the multipath echos rarely exceeds $3 \mathrm{~dB}$ in terrestrial digital broadcasting, $\tau_{\mathrm{DM}}$ can be used as a reliable blind replacement for $\tau_{M \infty}$.

\section{CONClusion}

The timing phase-offset recovery problem has been stated as a constrained channel optimization for a finite-length symbol-spaced equalizer with respect to the MSE of the equalizer output. A blind adaptive approach based on dispersion minimization can be used to achieve this optimality for a short equalizer. A geometrical argument is presented that allows comparison with the MMSE approach and issues of unimodality of the DM-solution have been addressed. The MSE performance of DM-timing has been investigated by comparing dispersion minimization timing offset with standard timing offsets. DM-timing offset achieves close MMSE performance for clustered multipath channels, regardless of the severity of the multipath. For sparse channels, the MSE performance of DM-timing degrades as the severity of the multipath increases but still achieves better performance than the conventional output energy maximization timing offset (see Figs. 5-7). 


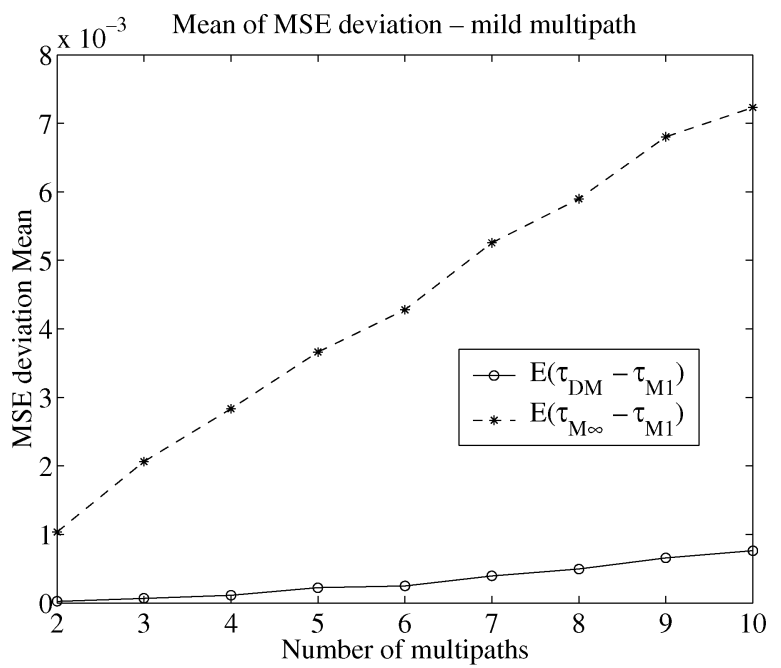

a)

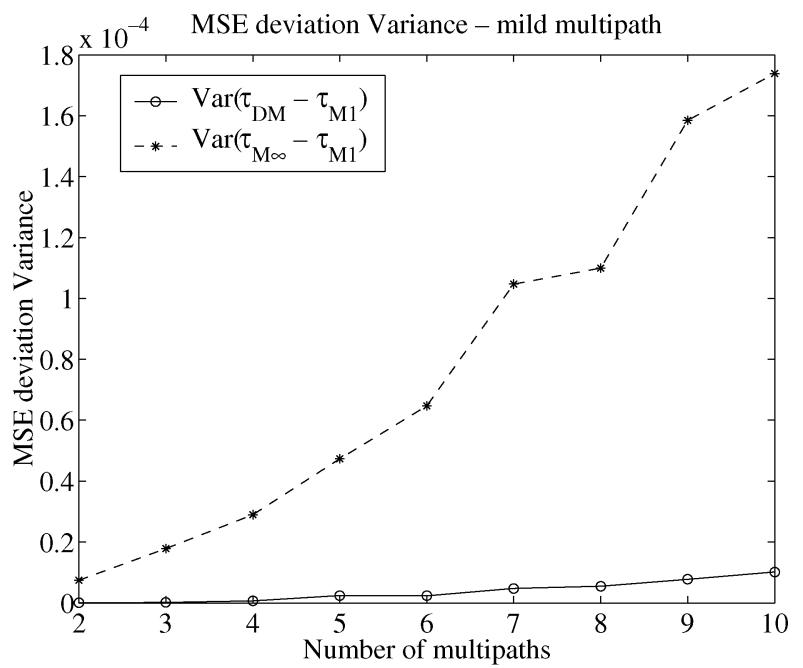

b)

Fig. 6. DM-timing and MSE-timing for mild multipath channels. (a) MSE deviation mean. (b) MSE deviation variance.

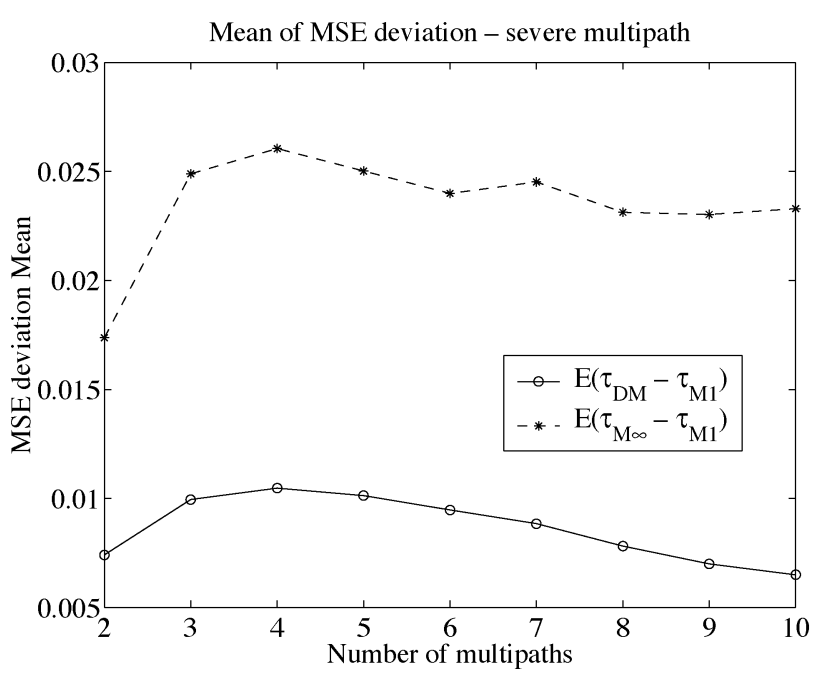

a)

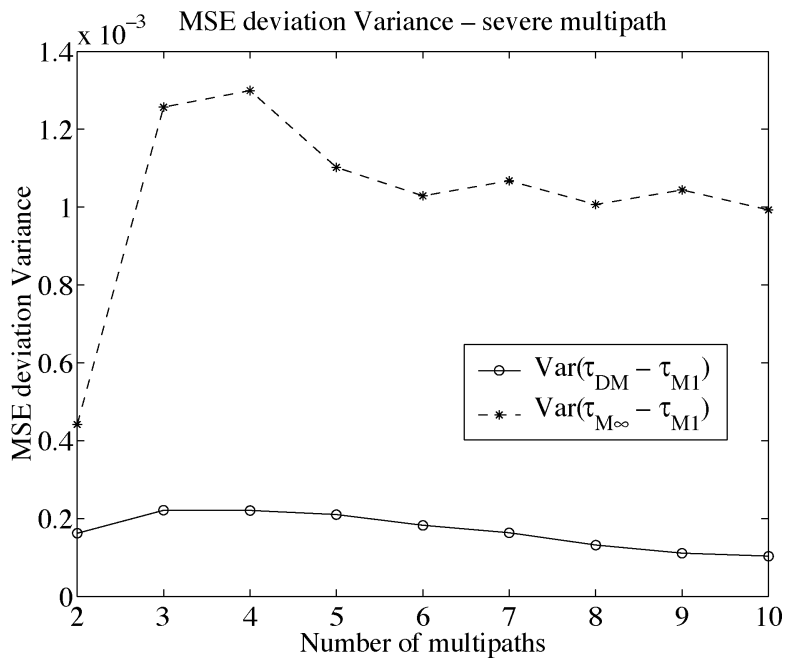

b)

Fig. 7. DM-timing and MSE-timing for severe multipath channels. (a) MSE deviation mean. (b) MSE deviation variance.

\section{APPENDIX}

\section{A. Proof of (22): Expansion of DM-Cost Function}

For a given parameter set $(\tau, \alpha)$, denote $\mathrm{h}=\alpha \mathrm{c}_{\tau}$. Then, the cost function (22) is written as

$$
\begin{aligned}
J(\tau, \alpha)= & E\left\{\left(\left|\mathrm{~h}^{T} s+\alpha w\right|^{2}-\gamma\right)^{2}\right\} \\
= & E\left(\left|\mathrm{~h}^{T} s\right|^{4}\right)+4 E\left\{\left|\mathrm{~h}^{T} \mathrm{~s}\right|^{2}|\alpha w|^{2}\right\} \\
& +E\left\{\left(\left(\mathrm{~h}^{T} \mathrm{~s}\right)^{*}\right)^{2}(\alpha w)^{2}\right\}+E\left\{\left(\mathrm{~h}^{T} \mathrm{~s}\right)^{2}\left(\alpha^{*} w^{*}\right)^{2}\right\} \\
& +E\left\{|\alpha w|^{4}\right\}-2 \gamma E\left\{\left|h^{T} s+\alpha w\right|^{2}\right\}+\gamma^{2} .
\end{aligned}
$$

First, from the fact $\left\{s_{i}\right\}$ is i.i.d.

$$
\begin{aligned}
E\left(\left|\mathrm{~h}^{T} \mathrm{~s}\right|^{4}\right)=m_{4} \sum_{i}\left|\mathrm{~h}_{i}\right|^{4} & +2 m_{2}^{2} \sum_{i \neq j}\left|h_{i}\right|^{2}\left|h_{j}\right|^{2} \\
& +\left|E\left(s_{n}^{2}\right)\right|^{2} \sum_{i \neq j} h_{i}^{2}\left(h_{j}^{*}\right)^{2}
\end{aligned}
$$

where $m_{2}$ is the second moment, and $m_{4}$ is the fourth moment. Since we assume $\{s\}$ is circular when it is complex

$$
\left|E\left(s_{n}^{2}\right)\right|^{2} \sum_{i \neq j} h_{i}^{2}\left(h_{j}^{*}\right)^{2}= \begin{cases}0, & \text { complex case } \\ m_{2}^{2} \sum_{i \neq j} h_{i}^{2} h_{j}^{2}, & \text { real case }\end{cases}
$$

Therefore

$$
\begin{aligned}
E\left(\left|h^{T} s\right|^{4}\right) & =m_{4} \sum_{i}\left|\mathrm{~h}_{i}\right|^{4}+\kappa_{w} m_{2}^{2} \sum_{i \neq j}\left|h_{i}\right|^{2}\left|h_{j}\right|^{2} \\
& =m_{4}\|\mathrm{~h}\|_{4}^{4}+\kappa_{w} m_{2}^{2}\left(\|\mathrm{~h}\|^{4}-\|\mathrm{h}\|_{4}^{4}\right) \\
& =\eta_{s} m_{2}^{2}\|\mathrm{~h}\|_{4}^{4}+\kappa_{w} m_{2}^{2}\|\mathrm{~h}\|^{4} .
\end{aligned}
$$

On the other hand

$$
\begin{aligned}
E\left\{\left(\left(\mathrm{~h}^{T} \mathrm{~s}\right)\right)^{2}\left(\alpha^{*} w^{*}\right)^{2}\right\} & =E\left\{\left(\mathrm{~h}^{T} \mathrm{~s}\right)^{2}\right\} E\left\{\left(\alpha^{*} w^{*}\right)^{2}\right\} \\
& =E\left(s^{2}\right) E\left(w^{*}\right)^{2} \sum_{i} h_{i}^{2}\left(\alpha^{*}\right)^{2} .
\end{aligned}
$$


Similarly

$$
\begin{aligned}
E\left(s^{2}\right) E\left(w^{*}\right)^{2} \sum_{i} & h_{i}^{2}\left(\alpha^{*}\right)^{2} \\
& = \begin{cases}0, & \text { complex case } \\
m_{2} \sigma_{w}^{2}\|\mathrm{~h}\|^{2}|\alpha|^{2}, & \text { real case. }\end{cases}
\end{aligned}
$$

Now, the cost function can be written as

$J(\tau, \alpha)=\eta_{s} m_{2}^{2}\|\mathrm{~h}\|_{4}^{4}+\kappa_{w} m_{2}^{2}\|\mathrm{~h}\|^{4}+2 \kappa_{w} m_{2} \sigma_{w}^{2}\|\mathrm{~h}\|^{2}|\alpha|^{2}$

$+E|w|^{4}\left(\sum_{k}\left|\beta_{k}\right|^{2}\right)^{2}-2 \gamma m_{2}\left(\|\mathrm{~h}\|^{2}+\sigma_{w}^{2} \sum_{k}\left|\beta_{k}\right|^{2}\right)+\gamma^{2}$.

Since $\sigma_{s}^{2}=1, m_{2}=1$ and $\gamma=\kappa_{s}$, and $\lambda=\sigma_{w}^{2}$. Furthermore, from the definition of kurtosis $\kappa_{w}$

$$
E\left\{|w|^{4}\right\}=\kappa_{w}\left(E\left\{|w|^{2}\right\}\right)^{2}=\kappa_{w} \lambda^{2} .
$$

Thus

$$
\begin{aligned}
J(\tau, \alpha)= & \eta_{s}\|\mathrm{~h}\|_{4}^{4}+\kappa_{w}\|\mathrm{~h}\|^{4}+2 \kappa_{w} \lambda\|\mathrm{h}\|^{2}|\alpha|^{2}+\kappa_{w} \lambda^{2}|\alpha|^{4} \\
& -2 \kappa_{s}\left(\|\mathrm{~h}\|^{2}+\lambda|\alpha|^{2}\right)+\kappa_{s}^{2} . \\
= & \alpha^{4}\left(\eta_{s}\left\|\mathrm{c}_{\tau}\right\|_{4}^{4}+\kappa_{w}\left(\left\|\mathrm{c}_{\tau}\right\|^{2}+\lambda\right)^{2}\right) \\
& -2 \kappa_{s} \alpha^{2}\left(\left\|\mathrm{c}_{\tau}\right\|^{2}+\lambda\right)+\kappa_{s}^{2} .
\end{aligned}
$$

\section{B. Proof of Theorem 1}

From (83) in Appendix A, the DM-cost function jointly with a scalar equalizer can be written as

$$
\begin{aligned}
J(\tau, \alpha)=\alpha^{4}\left(\eta_{s}\left\|\mathrm{c}_{\tau}\right\|_{4}^{4}+\kappa_{w}\right. & \left.\left(\left\|\mathrm{c}_{\tau}\right\|^{2}+\lambda\right)^{2}\right) \\
& -2 \alpha^{2}\left(\kappa_{s}\left\|\mathrm{c}_{\tau}\right\|^{2}+\lambda\right)+\kappa_{s}^{2} .
\end{aligned}
$$

Since the cost function is a quadratic of $\alpha^{2}$

$$
\alpha_{\mathrm{opt}}^{2}=\kappa_{s} \frac{\left\|\mathrm{c}_{\tau}\right\|^{2}+\lambda}{\eta_{s}\left\|\mathrm{c}_{\tau}\right\|_{4}^{4}+\kappa_{w}\left(\left\|\mathrm{c}_{\tau}\right\|^{2}+\lambda\right)^{2}}
$$

Now, $J$ can be written with respect to $\alpha_{\mathrm{opt}}^{2}$

$$
\begin{aligned}
J\left(\tau, \alpha_{\mathrm{opt}}\right) & =\kappa_{s}^{2}-\kappa_{s}^{2} \frac{\left(\left\|\mathrm{c}_{\tau}\right\|^{2}+\lambda\right)^{2}}{\eta_{s}\left\|\mathrm{c}_{\tau}\right\|_{4}^{4}+\kappa_{w}\left(\left\|\mathrm{c}_{\tau}\right\|^{2}+\lambda\right)^{2}} \\
& =\kappa_{s}^{2}-\kappa_{s}^{2} \frac{1}{\kappa_{w}+\eta_{s}\left(\frac{\left\|\mathrm{c}_{\tau}\right\|_{4}^{2}}{\left\|\mathrm{c}_{\tau}\right\|^{2}+\lambda}\right)^{2}} .
\end{aligned}
$$

Since $\eta_{s}$ is negative, we have

$$
\tau_{\mathrm{opt}}=\arg \max _{\tau} \frac{\left\|\mathbf{c}_{\tau}\right\|_{4}^{2}}{\left\|\mathbf{c}_{\tau}\right\|^{2}+\lambda} .
$$

\section{Proof of Theorem 2}

We use the following substitution:

$$
x=2 \pi \tau+\angle H_{4}(1), \quad 0 \leq x \leq 2 \pi
$$

so that we have

$$
I(x)=\frac{\sqrt{H_{4} \cos (x)+G_{4}}}{H_{2} \cos (x+\phi)+G_{2}}
$$

where $\phi=\angle H_{2}(1)-\angle H_{4}(1)$. Furthermore, we denote

$$
\begin{aligned}
& K(x)=H_{4} \cos (x)+G_{4} \\
& Q(x)=H_{2} \cos (x+\phi)+G_{2} .
\end{aligned}
$$

Since $I(x)>0$, the unimodality of $I(x)$ is equivalent to that of $I^{2}(x)$. Notice that $I^{2}(x)$ is bounded and periodic, and $I^{2}(0)=$ $I^{2}(2 \pi)$. Therefore, it is sufficient to show that the derivative of $I^{2}(x)$ has only two roots for $0<x \leq 2 \pi$ : One of those is the maximum, and the other one is the minimum. Consider the derivative

$$
\frac{d}{d x} I^{2}(x)=\frac{K^{\prime}(x) Q(x)-2 K(x) Q^{\prime}(x)}{Q^{3}(x)} .
$$

Because $Q(x)>0$, it is enough to show that the numerator in (93) has only two roots.

$$
\begin{aligned}
K^{\prime}(x) & Q(x)-2 K(x) Q^{\prime}(x) \\
= & 2 H_{2} K(x) \sin (x+\phi)-H_{4} Q(x) \sin x \\
= & {\left[\sin \phi 2 H_{2} K(x)\right] \cos x } \\
& -\left[H_{4} Q(x)-\cos \phi 2 H_{2} K(x)\right] \sin x .
\end{aligned}
$$

We want to apply the following trigonometry identity to the above equation:

$$
A \cos x+B \sin x=\sqrt{A^{2}+B^{2}} \sin \left(x+\tan ^{-1} \frac{A}{B}\right) .
$$

In order to do that, we need to check that the coefficient terms of $\sin x$ and $\cos x$ in (94) are nonzero for some $x$.

First, observe that

$$
\begin{aligned}
H_{4} Q & (x)-\cos \phi 2 H_{2} K(x) \\
= & H_{4}(G 2+H 2 \cos (x+\phi)) \\
& -\cos \phi 2 H_{2}\left(G_{4}+H_{4} \cos (x)\right) \\
= & G_{2} H_{4}-2 H_{2} G_{4} \cos \phi-H_{2} H_{4} \cos (x-\phi) \\
\geq & G_{2} H_{4}-2 H_{2} G_{4}-H_{2} H_{4}>0
\end{aligned}
$$

where, in the last step, we have used (40).

Second, when $\sin \phi=0$, then (94) collapses into the following sinusoid:

$$
I^{2}(x)=-\left[H_{4} Q(x)-\cos \phi 2 H_{2} K(x)\right] \sin x
$$


and has only two roots, since the coefficient is nonzero from (96). Therefore, we can assume $\sin \phi \neq 0$, which guarantees that for all $x \in[0,2 \pi]$, either

$$
\sin \phi 2 H_{2} K(x)>0 \text {, or } \sin \phi 2 H_{2} K(x)<0 \text {. }
$$

Now, we have (99), shown at the bottom of the page, where

$$
\varphi(x)=\tan ^{-1} \frac{\sin \phi 2 H_{2} K(x)}{H_{4} Q(x)-\cos \phi 2 H_{2} K(x)} .
$$

Since the coefficient of the sin term in (99) is nonzero, it is sufficient to show that the sin term

$$
\sin (x-\varphi(x))
$$

has only two roots for $0 \leq x<2 \pi$ in order to prove the theorem. Notice that $\varphi(x)$ is a continuous function of $\cos (x)$ and $\cos (x+$ $\phi)$; thus

$$
\varphi(0)=\varphi(2 \pi)
$$

When $x-\varphi(x)$ increases strictly monotonically for $0<x \leq$ $2 \pi$, i.e.,

$$
\frac{d}{d x}(x-\varphi(x))>0
$$

then

$$
-\varphi(0) \leq x-\varphi(x) \leq 2 \pi-\varphi(0)
$$

and $\cos (x-\varphi(x))$ has only two roots since

$$
\frac{d}{d x} \cos (x-\varphi(x))=-\sin (x-\varphi(x)) \frac{d}{d x}(x-\varphi(x)) \text {. }
$$

Therefore, we need to show that

$$
\frac{d}{d x}(x-\varphi(x))>0, \text { or } \frac{d}{d x} \varphi(x)<1 .
$$

We will show that $|(d / d x) \varphi(x)|<1$. Using the fact

$$
\frac{d}{d x} \tan ^{-1}(x)=\frac{1}{\left(1+x^{2}\right)}
$$

we have (108)-(112), shown at the bottom of the page. From (111) to (112), we used the following inequality from (40):

$$
\begin{aligned}
H_{4} Q(x)-2 H_{2} K(x)= & H_{4} H_{2} \cos (x+\phi)+H_{4} G_{2} \\
& -2 H_{2} H_{4} \cos (x)-2 H_{2} G_{4} \\
> & 2 H_{2} H_{4} .
\end{aligned}
$$

From (108) to (109), we utilized the following inequality:

$$
\left|\frac{\sin \phi}{a-b \cos \phi}\right| \leq \frac{1}{\sqrt{a^{2}-b^{2}}}
$$

\section{Proof of Lemma 1}

\section{Consider}

$$
\begin{aligned}
e^{-j 2 \pi \tau_{0} f} P(f) \star e^{-j 2 \pi \tau_{1} f} P(f) & \int_{-1}^{f+1} e^{-j 2 \pi \tau_{0} x} P(x) e^{-j 2 \pi \tau_{1}(f-x)} P(f-x) d x \\
= & e^{-j 2 \pi f \frac{\tau_{0}+\tau_{1}}{2}} \int_{-\frac{f}{2}-1}^{\frac{f}{2}+1} \cos \left(2 \pi x \frac{\tau_{0}-\tau_{1}}{2}\right) \\
& \times P\left(x-\frac{f}{2}\right) P\left(x+\frac{f}{2}\right) d x
\end{aligned}
$$

$$
I^{2}(x)=\left(\sqrt{\left(H_{4} Q(x)\right)^{2}-4 \cos \phi H_{2} H_{4} Q(x) K(x)+\left(2 H_{2} K(x)\right)^{2}}\right) \sin (x-\varphi(x))
$$

$$
\begin{aligned}
\left|\frac{d}{d x} \varphi(x)\right| & =\left|\frac{\sin \phi 2 H_{2} H_{4}\left(H_{4} Q(x) \sin (x)-H_{2} K(x) \sin (x+\phi)\right)}{\left(H_{4} Q(x)\right)^{2}+\left(H_{2} K(x)\right)^{2}-2 \cos \phi\left(H_{4} Q(x)\right)\left(2 H_{2} K(x)\right)}\right| \\
& \leq \frac{2 H_{2} H_{4}\left|H_{4} Q(x) \sin (x)-H_{2} K(x) \sin (x+\phi)\right|}{\sqrt{\left(\left(H_{4} Q(x)\right)^{2}+\left(2 H_{2} K(x)\right)^{2}\right)^{2}-\left(2\left(H_{4} Q(x)\right)\left(2 H_{2} K(x)\right)\right)^{2}}} \\
& \leq \frac{2 H_{2} H_{4}\left(H_{4} Q(x)+H_{2} K(x)\right)}{\left(H_{4} Q(x)\right)^{2}-\left(2 H_{2} K(x)\right)^{2}} \\
& \leq \frac{2 H_{2} H_{4}}{H_{4} Q(x)-2 H_{2} K(x)} \\
& <\frac{2 H_{2} H_{4}}{2 H_{2} H_{4}}=1 .
\end{aligned}
$$


From (116) and (117), we have used the change of variable $x \mapsto$ $x+f / 2$ and exploited the fact that $\sin (2 \pi x) P(x-f / 2) P(x+$ $f / 2)$ is an anti-symmetric function since $P(x-f / 2) P(x+$ $f / 2)$ is symmetric. Property $i$ ) follows from the fact that the integration portion in (117) is real valued. Again, from (117), we have

$$
\begin{aligned}
& \lim _{\left|\tau_{0}-\tau_{1}\right| \rightarrow 0}\left|e^{-j 2 \pi \tau_{0} f} P(f) \star e^{-j 2 \pi \tau_{1} f} P(f)\right| \\
& =\lim _{\left|\tau_{0}-\tau_{1}\right| \rightarrow 0} \mid \int_{-\frac{f}{2}-1}^{\frac{f}{2}+1} \cos \left(2 \pi x \frac{\tau_{0}-\tau_{1}}{2}\right) \\
& \quad \times P\left(x-\frac{f}{2}\right) P\left(x+\frac{f}{2}\right) d x \mid \\
& =\left|\int_{-\frac{f}{2}-1}^{\frac{f}{2}+1} P\left(x-\frac{f}{2}\right) P\left(x+\frac{f}{2}\right) d x\right| \\
& =P_{2}(f)
\end{aligned}
$$

which proves $i i)$. On the other hand, we have

$$
\begin{aligned}
& \lim _{\left|\tau_{0}-\tau_{1}\right| \rightarrow \infty}\left|e^{-j 2 \pi \tau_{0} f} P(f) \star e^{-j 2 \pi \tau_{1} f} P(f)\right| \\
&=\lim _{\left|\tau_{0}-\tau_{1}\right| \rightarrow \infty} \mid \int_{-\frac{f}{2}-1}^{\frac{f}{2}+1} e^{-j 2 \pi x \frac{\tau_{0}-\tau_{1}}{2}} \\
& \times P\left(x-\frac{f}{2}\right) P\left(x+\frac{f}{2}\right) d x \mid \\
& \leq \lim _{\left|\tau_{0}-\tau_{1}\right| \rightarrow \infty}\left|\int_{-\frac{f}{2}-1}^{\frac{f}{2}+1} e^{-j 2 \pi x \frac{\tau_{0}-\tau_{1}}{2}} d x\right| \\
&=\lim _{\left|\tau_{0}-\tau_{1}\right| \rightarrow \infty}\left|\left(1+\frac{f}{2}\right) \operatorname{sinc}\left[\left(\tau_{0}-\tau_{1}\right)\left(1+\frac{f}{2}\right)\right]\right| \\
&=0
\end{aligned}
$$

where in (122), we used the fact that $P$ is non-negative. This proves iii).

\section{E. Proof of Inequality (35)}

Since $H_{n}(f)$ can be written as a sum of $n$-convolutions drawn from combinations of $\left\{P(f) \rho_{k} e^{-j 2 \pi \tau_{k} f} \mid k=0, \cdots, N_{c}-1\right\}$

$$
\begin{aligned}
H_{n}(f) & =\sum_{\substack{k_{i} \in\left\{0, \ldots, N_{c}-1\right\} \\
\star P(f) \rho_{k_{2}} e^{-j 2 \pi \tau_{k_{2}} f} \star \cdots \star P(f) \rho_{k_{n}} e^{-j 2 \pi \tau_{k_{n}} f}}} P(f) \rho_{k_{1}} e^{-j 2 \pi \tau_{k_{1}} f}
\end{aligned}
$$

we have

$$
\begin{aligned}
& \left.\mid H_{n}(f)\right)\left|\leq \sum_{k_{i} \in\left\{0, \ldots, N_{c}-1\right\}}\right| P(f) \rho_{k_{1}} e^{-j 2 \pi \tau_{k_{1}} f} \\
& \star P(f) \rho_{k_{2}} e^{-j 2 \pi \tau_{k_{2}} f} \star \cdots \star P(f) \rho_{k_{n}} e^{-j 2 \pi \tau_{k_{n}} f} \mid .
\end{aligned}
$$

In order to show that

$$
\begin{aligned}
& \mid P(f) \rho_{k_{1}} e^{-j 2 \pi \tau_{k_{1}} f} \star P(f) \rho_{k_{2}} e^{-j 2 \pi \tau_{k_{2}} f} \\
& \quad \star \cdots \star P(f) \rho_{k_{n}} e^{\left.-j 2 \pi \tau_{k_{n}} f\right)} \mid \leq P_{n}(f) \prod \rho_{k_{i}}
\end{aligned}
$$

consider a two-term-convolution case first:

$$
\begin{aligned}
& \left|P(f) \rho_{k} e^{-j 2 \pi \tau_{k} f} \star P(f) \rho_{m} e^{-j 2 \pi \tau_{m} f}\right| \\
& \quad \leq\left|P(f) \rho_{k} e^{-j 2 \pi \tau_{k} f}\right| \star\left|P(f) \rho_{m} e^{-j 2 \pi \tau_{m} f}\right| \\
& \quad=P(f) \rho_{k} \star P(f) \rho_{m} \\
& \quad=P_{2}(f)\left(\rho_{k} \rho_{m}\right) .
\end{aligned}
$$

In (128), we used the Schwartz Inequality, and in (129), we used the fact that $P(f)$ and $\rho_{k}, \rho_{m}$ are non-negative real.

Applying this inequality inductively for each $n$-convolution term, inequality (35) can be obtained.

\section{REFERENCES}

[1] J. Proakis, Digital Communications. New York: McGraw-Hill, 1995.

[2] F. Guglielmi, C. Luschi, and A. Spalvieri, "Joint clock recovery and baseband combining for the diversity radio channel," IEEE Trans. Commun., vol. 44, no. 1, pp. 114-117, Jan. 1996.

[3] H. Kobayashi, "Simultaneous adaptive estimation and decision algorithm for carrier-modulated data transmission systems," IEEE Trans. Commun., vol. COM-19, pp. 268-280, Jun. 1971.

[4] D. N. Godard, "Passband timing recovery in all-digital modem receiver," IEEE Trans. Commun., vol. COM-26, pp. 517-523, May 1978.

[5] R. D. Giltin and S. B. Weinstein, "Fractionally spaced equalization: an improved digital transversal equalizer," Bell Syst. Tech. J., pp. 301-321, 1981.

[6] W. F. Schreiher, "Advanced television systems for terrestrial broadcasting: some problems and proposed solutions," Proc. IEEE, vol. 83, no. 6, pp. 958-981, Jun. 1995.

[7] W. Chung, J. Balakrishnan, W. Sethares, and C. R. Johnson, Jr., "Timing recovery based on dispersion minimization," in Proc. Conf. Inform. Sci. Syst., Mar. 2001.

[8] D. L. Lyon, "Timing recovery in synchronous equalized data communication," IEEE Trans. Commun., vol. 23, no. 2, pp. 269-274, Feb. 1975.

[9] J. E. Mazo, "Optimum timing phase for an infinite equalizer," Bell Syst. Tech. J., pp. 189-201, Jan. 1975.

[10] W. Chung, "Blind Parameter Estimation for Data Acquisition in Digital Communication Systems," Ph.D. dissertation, Cornell Univ., Ithaca, NY, Aug. 2002.

[11] C. R. Johnson, Jr., P. Schniter, T. J. Endres, J. D. Behm, D. R. Brown, and R. A. Casas, "Blind equalization using the constant modulus criterion: a review," Proc. IEEE, vol. 86, pp. 1927-1950, Oct. 1998.

[12] J. R. Treichler, C. R. Johnson, Jr., and M. G. Larimore, Theory and Design of Adaptive Filters. Englewood Cliffs, NJ: Prentice-Hall, 2001.

[13] ATSC Digital Television Standard (Tech. Rep. Doc. A/53), Advanced Television Systems Committee. (2000, Mar.). www.tsc.org [Online]

[14] H. Holma and A. Toskala, WCDMA for UMTS. New York: Wiley, 2001.

[15] H. Zeng, L. Tong, and C. R. Johnson, Jr., "Relationships between the constant modulus and Wiener receivers," IEEE Trans. Inf. Theory, vol. 44, no. 4, pp. 1523-1538, Jul. 1998.

[16] P. Schniter and C. R. Johnson, Jr., "Bounds for the MSE performance of constant modulus estimators," IEEE Trans. Inf. Theory, vol. 47, no. 7, pp. 2544-2560, Nov. 2000.

[17] M. Gu and L. Tong, "Geometrical characterization of constant modulus receivers," IEEE Trans. Signal Processing, vol. 47, no. 10, pp. 2745-2756, Oct. 1999.

[18] S. Y. Kung, "Independent component analysis in hybrid mixture: KuicNet learning algorithms and numerical analysis," in Proc. Int. Symp. Multimedia Inform. Process., Dec. 1997, pp. 368-381. 


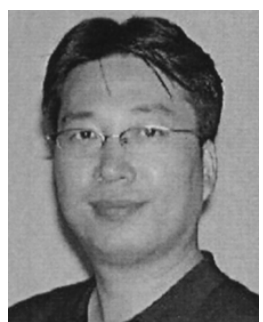

Wonzoo Chung received the B.A. degree in mathematics from Korea University, Seoul, Korea, and the M.S. and Ph.D. degrees in electrical engineering from Cornell University, Ithaca, NY.

He is currently with Dotcast, Inc., Kent, WA, as Senior System Architect, working on research and development of signal processing technologies for the digital data broadcasting system. His research interests include blind and adaptive aspects of digital signal processing for telecommunication systems.

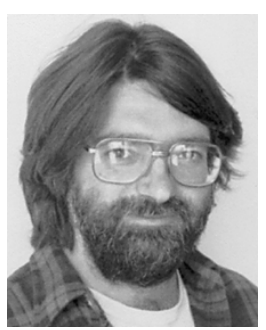

William A. Sethares received the B.A. degree in mathematics from Brandeis University, Waltham, MA and the M.S. and Ph.D. degrees in electrical engineering from Cornell University, Ithaca, NY.

$\mathrm{He}$ has worked at the Raytheon Company, Waltham, as a Systems Engineer and is currently a Professor with the Department of Electrical and Computer Engineering, University of Wisconsin, Madison. His research interests include adaptation and learning in signal processing, communications, and acoustics. He is the author of Tuning, Timbre, Spectrum, Scale (New York: Springer 1998) and of Telecommunication Breakdown (Englewood Cliffs, NJ: Prentice-Hall 2003).

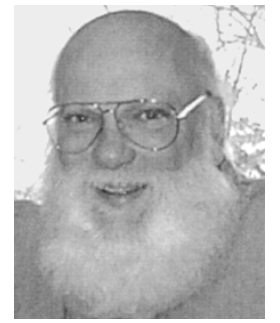

C. Richard Johnson, Jr. (F'89) was born in Macon, GA, in 1950. He received the Ph.D. degree in electrical engineering with minors in engineering-economic systems and art history from Stanford University, Stanford, CA, in 1977.

$\mathrm{He}$ is currently a Professor of electrical and computer engineering and a member of the Graduate Field of Applied Mathematics at Cornell University, Ithaca, NY. Since 1990, he has held visiting appointments at Stanford University; the University of California-Berkeley; Chalmers University of Technology, Gothenburg, Sweden; the Technical University of Vienna, Vienna, Austria; National Polytechnic Institute of Grenoble, Grenoble, France; and the Australian National University, Canberra, Australia. During that period, his primary research interest has been blind adaptive fractionally spaced linear and decision feedback equalization for intersymbol and structured multiuser interference removal from single and multicarrier communication systems. The current broadband adaptive receiver design project of his research group at Cornell is described at http://bard.ece.Cornell.edu/. The group's activity is currently supported by the National Science Foundation, Applied Signal Technology, and Texas Instruments.

Dr. Johnson was elected a Fellow of the IEEE "for contributions to adaptive parameter estimation theory with applications in digital control and signal processing" in 1989. 